\title{
Mixed quantum-classical dynamics of an amide-I vibrational excitation in a protein $\alpha$-helix
}

\author{
Holly Freedman, ${ }^{1}$ Paulo Martel, ${ }^{2}$ and Leonor Cruzeiro ${ }^{1,3}$ \\ ${ }^{1}$ CCMAR, FCT, University of Algarve, Campus de Gambelas, Faro, Portugal \\ ${ }^{2}$ Center for Structural and Molecular Biomedicine, University of Algarve, Campus de Gambelas, Faro, Portugal \\ ${ }^{3}$ Department of Physics, FCT, University of Algarve, Campus de Gambelas, Faro, Portugal \\ (Received 28 September 2010; revised manuscript received 8 November 2010; published 24 November 2010)
}

\begin{abstract}
Adenosine triphosphate (ATP) is known to be the main energy currency of the living cell, and is used as a coenzyme to generate energy for many cellular processes through hydrolysis to adenosine diphosphate (ADP), although the mechanism of energy transfer is not well understood. It has been proposed that following hydrolysis of the ATP cofactor bound to a protein, up to two quanta of amide-I vibrational energy are excited and utilized to bring about important structural changes in the protein. To study whether, and how, amide-I vibrational excitations are capable of leading to protein structural changes, we have added components arising from quantum-mechanical amide-I vibrational excitations to the total energy and force terms within a moleculardynamics simulation. This model is applied to helical deca-alanine as a test case to investigate how its dynamics differs in the presence or absence of an amide-I excitation. We find that the presence of an amide-I excitation can bias the structure toward a more helical state.
\end{abstract}

DOI: $10.1103 /$ PhysRevB.82.174308

PACS number(s): 34.50.Ez, 87.10.Tf, 87.15.bd

\section{INTRODUCTION}

Adenosine triphosphate (ATP) binds to many proteins and upon hydrolysis to adenosine diphosphate (ADP) leads to an altered conformational state of the protein, resulting in protein activation or deactivation. This reaction releases about $8-12 \mathrm{kcal} / \mathrm{mol}$ of energy, which is on the order of magnitude of vibrational excitations. However it is not understood how the energy released is channeled to contribute to the protein's conformational change. While vibrational excitations are most likely excited, not only are their energies small by comparison with other molecular interactions involved in protein dynamics, but also isolated vibrations have extremely short lifetimes of on the order of $10^{-13}$ s. On the other hand, according to the second law of thermodynamics, any work that is performed by the protein requires the utilization of the energy released by hydrolysis.

Davydov has formulated a hypothesis about how this energy transfer might take place. He noted that the energy provided by ATP hydrolysis is equivalent to approximately two quanta of amide-I vibrational energy. The amide-I mode consists mainly of stretching the $\mathrm{C}=\mathrm{O}$ bond, with an additional component from the $\mathrm{N}-\mathrm{H}$ bend. This mode seems an attractive candidate for vibrational energy storage since it is generally isolated and decoupled from other modes. Davydov proposed that vibrational self-trapping in the form of solitons may serve as a means of stable energy transfer in protein $\alpha$-helices. ${ }^{1,2}$

In an $\alpha$-helix, hydrogen bonding occurs between each backbone $\mathrm{C}=\mathrm{O}$ and the backbone $\mathrm{N}-\mathrm{H}$ group four residues ahead of it in the peptide chain; thus three chains of hydrogen-bonded groups run along its length. When an amide-I mode is excited in one peptide group, it results in the alteration of some of the hydrogen bond lengths in this lattice. This deformation leads to localization, or what is known as self-trapping, of the vibrational state. ${ }^{3}$

It was originally thought by Davydov that vibrational excitations are trapped as solitons for longer periods but experi- mental investigations of long-lived vibrational states have concluded that lifetimes are no longer than a few picoseconds. ${ }^{4,5}$ Thus the issue remains of how relatively short-lived vibrational excitations can perform work.

The dynamics of propagation of an amide-I state has previously been modeled by propagating vibrational states over system coordinates that had been precalculated by molecular-dynamics (MD) simulation. For example, such an approach was used to estimate energy transfer from water molecules to a prion described by a fully atomic force field, ${ }^{6}$ and has also been applied to calculate the linear absorption spectrum of an $\alpha$-helix. ${ }^{7}$ Here we include the bidirectional coupling between the process of vibrational energy propagation and the dynamics of the system, by adding an interaction energy term, coupling the classical dynamics with the amide-I excitation, to the potential driving an MD simulation. In this way not only does the protein environment affect the amide-I state but also the amide-I state affects protein dynamics.

Using this mixed-quantum classical MD implementation, we investigate whether amide-I excitations are capable of inducing a conversion of a disordered-helical peptide into a helical one. This hypothesis arose from the observation that a helical structure might be expected to allow for optimal interaction of all amide-I moieties, along a chain, with lattice phonons.

\section{METHODS}

The Davydov/Scott Hamiltonian $\hat{H}=\hat{H}_{e x}+\hat{H}_{a t}+\hat{H}_{\text {int }}$ describes the total energy of the protein system. ${ }^{6}$ This includes the second-quantized operator,

$$
H_{e x}=\varepsilon \sum_{n=1}^{N} a_{n}^{+} a_{n}+\sum_{n=1}^{N} \sum_{\substack{m=1 \\ m \neq n}}^{N} J_{n m}\left(a_{n}^{+} a_{m}+a_{m}^{+} a_{n}\right)
$$


describing the vibrational excitation, where $a_{n}^{+}$and $a_{n}$ are the usual creation and annihilation operators for an amide-I excitation on site $n, N$ is the number of amide-I sites, $\varepsilon$ is the vibrational frequency of the isolated amide-I mode, and $J_{n m}$ are dipole-dipole coupling parameters. $\hat{H}_{a t}$ is the operator associated with atomic displacements given by the classical force-field potential, which takes into account both bonded and nonbonded energy terms depending upon the full atomic composition of a protein. ${ }^{8,9}$ Finally,

$$
\hat{H}_{\text {int }}=\chi \sum_{n=1}^{N}\left[\left(\left|\vec{R}_{n}^{O}-\vec{R}_{m}^{N}\right|-d_{e q}\right) \hat{a}_{n}^{+} \hat{a}_{n}\right]
$$

is the interaction between the vibrational excitations and the atomic displacements, and depends on the parameter of nonlinearity $\chi=d \varepsilon / d\left(\left|\vec{R}_{n}^{O}-\vec{R}_{m}^{N}\right|\right)$. Here $d_{e q}$ denotes the equilibrium length of this hydrogen bond, set equal to $0.3 \mathrm{~nm}$ according to the force field. ${ }^{10}$ A positive value of $\chi$ means that an amide-I vibration favors compression of the hydrogen bond between the carbonyl oxygen atom on site $n$ at position $\vec{R}_{n}^{O}$ and the amine nitrogen atom on site $m$ at position $\vec{R}_{m}^{N}$; for a negative value, expansion of the hydrogen bond is favored instead. Estimates of $\chi$ based on experiments range from 35 to $62 \mathrm{pN}$, while a high degree of discrepancy exists among those determined computationally, which range from -60 to $26 \mathrm{pN},{ }^{11}$ since it is difficult to determine a suitable basis set to describe the very elastic hydrogen bond.

The mixed quantum-classical approach, which we use to determine the motion of the protein system, was implemented as a modification of the GROMACS program. ${ }^{8,9}$ GROMACS (Refs. 8 and 9) is a classical MD program, which solves Newton's equations of motion for atoms modeled as masses associated with point charges, interacting by springlike bonded interactions (bond stretching, angle bending, and torsions) as well as by nonbonded interactions (electrostatic and van der Waals interactions). This approach consists of coupling the classical Langevin equations of motion for the atoms in the protein, which are described by the AMBER potential, ${ }^{10,12}$ to the time-independent Schrödinger equation for the amide-I propagation, in order to satisfy both the classical statistics of the atomic motions and the quantum statistics of the amide-I states, as explained in detail in the Appendix. The integration of these mixed quantum-classical equations is comprised of three parts. Starting with a set of atomic coordinates, we first find the states of the amide-I vibrational excitation. These eigenstates are expressed in terms of a basis set consisting of states $|n\rangle$ for which oscillator $n$ is excited and all others are in the ground state: $\left|\Psi_{j}\right\rangle=\sum_{n=1}^{N} \varphi_{j n}|n\rangle$. They are determined as the eigenfunctions of the Hamiltonian corresponding to the system of coupled harmonic oscillators, parametrically dependent on the classical coordinates $u_{n}=\left|\vec{R}_{n}^{O}-\vec{R}_{m}^{N}\right|-d_{e q}$,

$$
\begin{aligned}
\hat{H}|\boldsymbol{\psi}\rangle & =\left[\begin{array}{cccc}
\varepsilon+\chi u_{1} & J_{12} & J_{13} & \cdots \\
J_{21} & \varepsilon+\chi u_{2} & & \vdots \\
\vdots & & \ddots & \\
& & & \varepsilon+\chi u_{N}
\end{array}\right]\left[\begin{array}{c}
\varphi_{1} \\
\varphi_{2} \\
\vdots \\
\varphi_{N}
\end{array}\right] \\
& =E\left[\begin{array}{c}
\varphi_{1} \\
\varphi_{2} \\
\vdots \\
\varphi_{N}
\end{array}\right]=E|\boldsymbol{\psi}\rangle .
\end{aligned}
$$

Dipole-dipole coupling parameters $J_{n m}$ are determined as interactions between transition dipoles, using the classical expression for the interaction between two dipoles,

$$
J_{n, m}=\frac{1}{4 \pi \varepsilon_{0} k} \frac{\left|\vec{\mu}_{n}\right|\left|\vec{\mu}_{m}\right|}{R_{n, m}^{3}}\left[\vec{e}_{n} \cdot \vec{e}_{m}-3\left(\vec{v}_{n, m} \cdot \vec{e}_{n}\right)\left(\vec{v}_{n, m} \cdot \vec{e}_{m}\right)\right]
$$

Here $\mu_{n}$ is the transition dipole moment of site $n$ with unit direction vector $\vec{e}_{n}$ and magnitude $0.30 \mathrm{D}, R_{n, m}$ and $\vec{v}_{n, m}$ are, respectively, the magnitude and unit direction vectors corresponding to the vector pointing from the center of one dipole to the center of the other, $k$ is the dielectric constant, and $\varepsilon_{0}$ is the vacuum permittivity constant. The direction of the transition dipole moment is taken to be at a $17^{\circ}$ angle to the $\mathrm{CO}$ bond in the direction of the $\mathrm{CN}$ bond, in accordance with experimental findings obtained for $\alpha$-helices. ${ }^{13,14}$ The associated eigenvalues $E$ are the energies of these $N$ states.

The second part of the integration consists of the propagation of the amide-I excitation into one of the $N$ states. The state with the best overlap with the previous state is chosen, and then a Metropolis step is performed to decide whether to retain the previous state or propagate into the new state. ${ }^{15}$ Specifically, the product of the squared overlap between the two states with the Boltzmann factor $\exp \left[-\left(E_{f}-E_{i}\right) / k_{B} T\right], k_{B}$ being the Boltzmann constant and $E_{f}$ and $E_{i}$ the energies of the new and previous states, respectively, is computed. If this value is less than a random number chosen between 0 and 1 , then the move is accepted. Otherwise the eigenstate that has the greatest probability in the same site as the previous state is chosen.

Finally, in the third part of our implementation, the MD equations of motion of the classical part of the system,

$$
M_{n} \frac{d^{2} R_{n}}{d t^{2}}=-\frac{\partial H_{\mathrm{int}}}{\partial R_{n}}-\frac{\partial H_{a t}}{\partial R_{n}}+F_{n}(t)-\Gamma \frac{d R_{n}}{d t},
$$

where $M_{n}$ is the mass of atom $n, F_{n}$ are stochastic forces, and $\Gamma \frac{d u_{n}}{d t}$ are damping terms, are integrated. Since the interaction energy term $H_{\text {int }}$ shown in Eq. (2), which represents the influence of the amide-I excitation on the classical motion, depends explicitly upon the positions of the hydrogenbonded $\mathrm{N}$ and $\mathrm{O}$ atoms, for these atoms force terms given by 


$$
-\frac{\partial H_{\text {int }}}{\partial R_{n}^{O}}=-\left|\varphi_{n}\right|^{2} \chi \frac{\vec{R}_{n}^{O}-\vec{R}_{m}^{N}}{\left|\vec{R}_{n}^{O}-\vec{R}_{m}^{N}\right|}, \quad-\frac{\partial H_{\text {int }}}{\partial R_{n}^{N}}=-\left|\varphi_{n}\right|^{2} \chi \frac{\vec{R}_{n}^{N}-\vec{R}_{m}^{O}}{\left|\vec{R}_{n}^{O}-\vec{R}_{m}^{N}\right|},
$$

are included in Eq. (5). All other atoms are propagated using standard molecular dynamics only, i.e., the last three terms in Eq. (5).

An explanation of our choice of the semiclassical approach just described to address the problem of introducing thermal baths to mixed quantum-classical systems is given in the Appendix. Equations (3)-(6) have previously been applied to a lattice model at finite temperature for which it was shown to predict lattice displacements agreeing well with those derived from a fully quantum-mechanical model $;{ }^{16}$ in addition absorption spectra have been calculated with features qualitatively similar to the experimental ones. ${ }^{7,15,17}$

We chose helical deca-alanine as a test case due to its simple repeating sequence, and the fact that it has been the subject of previous studies and is known to display some level of helicity. ${ }^{18}$ We first created helical deca-alanine (Ace- $\mathrm{Ala}_{10}-\mathrm{NH}_{2}$ ) by setting all $\phi / \psi$ dihedral angles to $-57.8^{\circ} /-47.0^{\circ}$. The leap and antechamber modules of the MD program AMBER9 (Ref. 12) were used to perform the initial setup, for convenience. Deca-alanine was parametrized using the AMBER99SB force field ${ }^{10}$ this force field is an update of the AMBER99 force field ${ }^{19,20}$ in which parameters were adjusted to give a better representation of secondary structure. The peptide was solvated by a periodic box of transferable intermolecular potential 3 point (TIP3P) water ${ }^{21}$ extending $12 \AA$ in the $x, y$, and $z$ directions. Structures were converted from AMBER to GROMACS-style coordinate and topology formats using the amb2gmx perl script, ${ }^{22}$ and simulations were then performed with GROMACS V4. ${ }^{8,9}$ The LINCS algorithm ${ }^{23}$ was used for bond constraints in all simulations, and we used a nonbonded cutoff of $12 \AA$. The particle mesh Ewald (PME) method was employed to calculate long-range electrostatics.

The system was equilibrated by running MD for $200 \mathrm{ps}$ at a temperature of $300 \mathrm{~K}$; a 2 fs time step was used for equilibration as well as throughout all following simulations. When the simulation is then restarted at $0 \mathrm{~K}$ this effectively anneals the system into a lower energy structure with less helical character, so that the deca-alanine assumes the structure of a disordered helix. Classical MD simulation was then carried out to bring the system to a temperature of $300 \mathrm{~K}$ over a 4 ps time period. Production runs used for analysis consist of the following 4 ps trajectories, run with periodic boundary conditions at a temperature of $300 \mathrm{~K}$ and at constant pressure. For the sake of statistical analysis, we obtained 20 similar trajectories by varying the seed of the random number generator used to obtain starting velocities for the first 4 ps of classical MD simulations. The simulation time of 4 ps was chosen based upon the fact that twodimensional-IR experiments performed by Hamm and coworkers have indicated that an amide-I excitation has a lifetime of up to about $2 \mathrm{ps}$ in an $\alpha$-helix; this is the time required for the bleach of the anomalous band to recover after being selectively excited. ${ }^{4,24}$

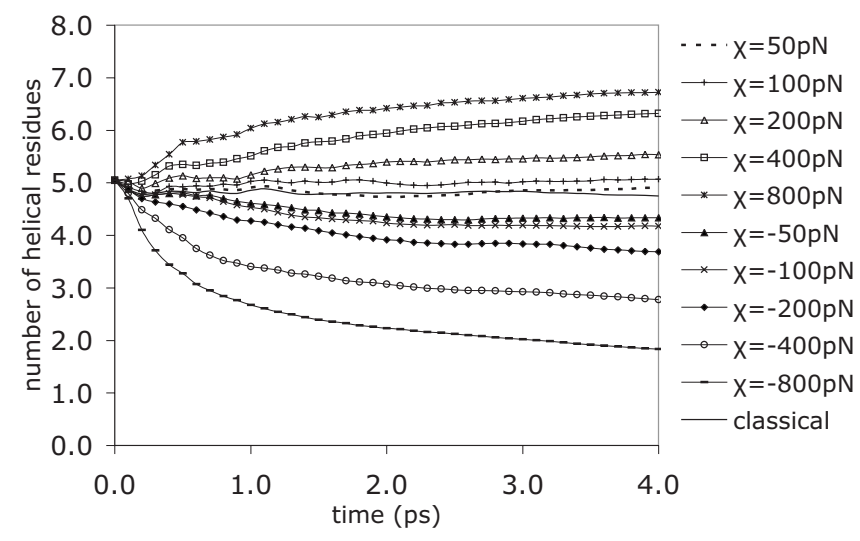

FIG. 1. Cumulative average of the number of helical residues as a function of simulation time (ps) determined by the $g_{-}$helix GROMACS analysis tool (Refs. 8 and 9).

\section{RESULTS}

Mixed quantum-classical simulations were performed using a range of values of the parameter $\chi$, namely, positive and negative values of $50,100,200,400$, and $800 \mathrm{pN}$. In addition, classical simulations were performed, for which the amide-I vibrational excitation was not included.

The number of residues corresponding to the longest helical segment was determined for each time point along the trajectory using the GROMACS analysis tools do_dssp and g_helix. The first of these, do_dssp, assigns secondary structure by identifying patterns of hydrogen bonding using the DSSP (Ref. 25) algorithm. The second program, g_helix, determines helicity based upon both hydrogen bonds and $\phi / \psi$ angles. ${ }^{8,9}$ Values were averaged over the 20 trajectories which were generated, and cumulative averages are plotted as a function of time in Figs. 1 and 2.

Interestingly the results confirm our hypothesis that the amide-I excitation leads to changes in the degree of helicity of the peptide. In the classical simulation, the number of helical residues calculated by either of the two methods remains roughly constant with time. However in the mixed quantum-classical simulations, this number either increases or decreases with time, depending on the sign of $\chi$. Positive

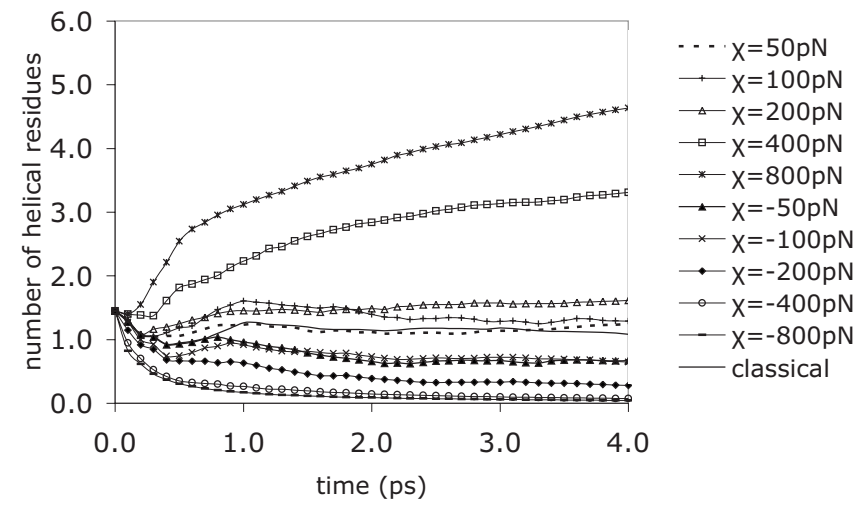

FIG. 2. Cumulative average of the number of helical residues as a function of simulation time (ps) determined by the do_dssp GROMACS analysis tool (Refs. 8 and 9). 


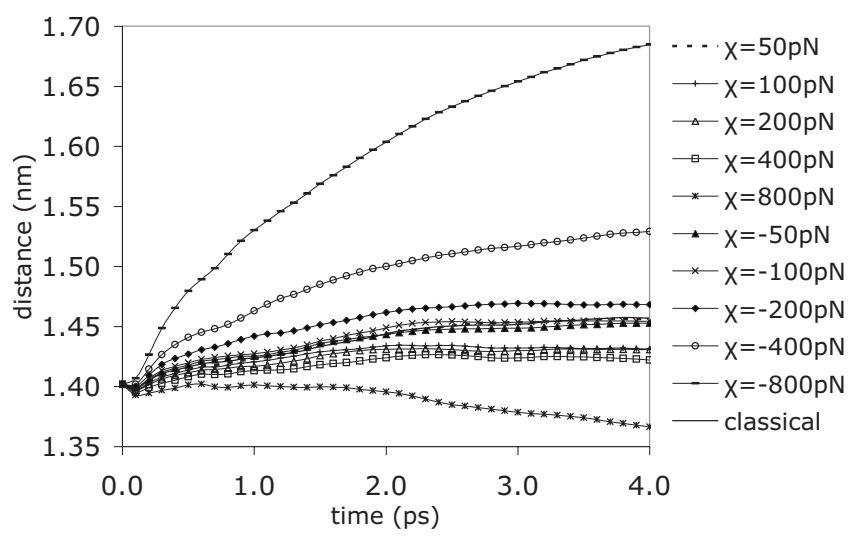

FIG. 3. Cumulative average of the minimum distance from the $\mathrm{N}$-terminal Ace group to the C-terminal $\mathrm{NH}_{2}$ group as a function of simulation time (ps) determined by the $g \_$mindist GROMACS analysis tool (Refs. 8 and 9).

values of $\chi$ bias the peptide structure toward a more helical form, and negative values show the opposite effect.

In Fig. 3 we plot distances from the acetyl group at the $\mathrm{N}$-terminal end of the peptide to the $\mathrm{NH}_{2}$ cap at the $\mathrm{C}$-terminal end. Contraction of the peptide is evident for positive values of $\chi$, due to the compression of hydrogen bonds described by Eq. (2). Likewise there is a reverse trend toward extension of the peptide when $\chi$ is negative.

We also analyzed the degree of localization of the amide-I excitation. This can be described by a parameter known as localization, given by $L_{j}=\sum_{n=1}^{N}\left|\varphi_{j n}\right|^{4}$, where $\left\{\varphi_{j n}\right\}$ are the coefficients in the expansion of eigenstate $j$ in terms of the states $|n\rangle$ for which oscillator $n$ is excited and all other oscillators are in the ground state. In this way, localization equals 1 for a completely localized state corresponding to a single nonzero coefficient, and equals $1 / N$ for a completely delocalized state for which all coefficients in the expansion are equal. Computed values of localization, averaged over time and over each of our 20 data sets, range from 0.22 to 0.24 depending on the value of $\chi$; these are tabulated in Table I. As the magnitude of $\chi$ is increased, states become more localized for negative $\chi$, but they become less localized

TABLE I. Average localization. The second column gives the standard deviation over the 20 data sets.

\begin{tabular}{rcc}
\hline \hline $\begin{array}{c}\chi \\
(\mathrm{pN})\end{array}$ & Localization & Stdev \\
\hline-800 & 0.243 & 0.019 \\
-400 & 0.234 & 0.021 \\
-200 & 0.232 & 0.012 \\
-100 & 0.233 & 0.010 \\
-50 & 0.236 & 0.008 \\
50 & 0.233 & 0.011 \\
100 & 0.230 & 0.009 \\
200 & 0.230 & 0.008 \\
400 & 0.220 & 0.009 \\
800 & 0.223 & 0.011 \\
\hline \hline
\end{tabular}
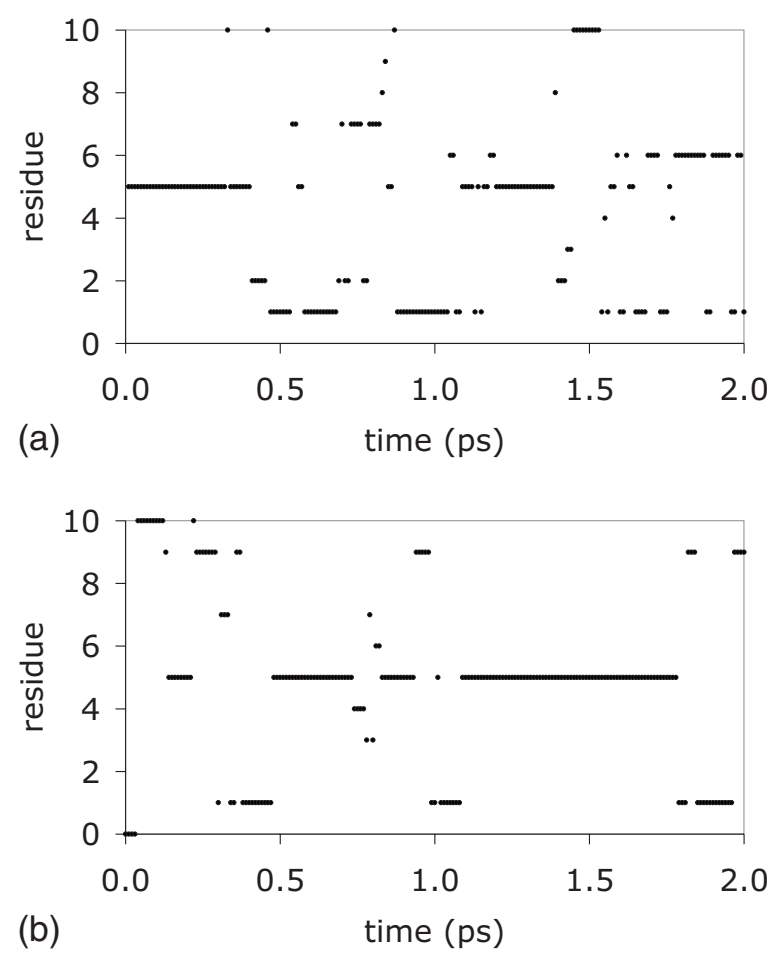

FIG. 4. Residue number at which the amide-I excitation is primarily located, i.e., for which the coefficient in the expansion in terms of basis functions localized on individual residues is largest, plotted as a function of simulation time (ps) for trajectories corresponding to (a) $\chi=800 \mathrm{pN}$ and (b) $\chi=100 \mathrm{pN}$.

for positive $\chi$. The former trend is what is expected to occur if structure remains static since decreasing the magnitude of $\chi$ increases the relative magnitude of off-diagonal elements of the Hamiltonian matrix in Eq. (3). However increased helicity leads to higher symmetry and thus also to more delocalization, explaining the trend for positive values of $\chi$.

We determined maximum values of the probability amplitude of the amide-I excitation on any given site ranging from 0.4 to 0.9 , by comparison with the value of 0.2 found in the study of a protein $\alpha$-helix performed by Brizhik et al. ${ }^{26}$ This smaller localization is most likely due to the use of a perfectly helical structure and the neglect of thermal noise in that study.

In Fig. 4(a), we plot the residue number corresponding to the highest content of vibrational excitation for a typical trajectory computed for $\chi=800 \mathrm{pN}$; only the first 2 ps are shown for better resolution. It can be seen that the amide-I excitation is transmitted throughout the entire peptide during the 2 ps simulation. There is, in general, a tendency for the excitation to be located on residue 5, i.e., at the middle of the peptide, or at one of the two ends. This is most likely due to greater flexibility of middle and end residues, as may be seen in the plot of root-mean-square fluctuation (RMSF) of $\alpha$ carbon atoms shown in Fig. 5. For this trajectory, $\langle L\rangle=0.21$; when localization is larger, the amide-I excitation remains at the same site for longer periods of time, as in the trajectory shown in Fig. 4(b) for which $\chi=100 \mathrm{pN}$ and $\langle L\rangle=0.25$.

The dynamical trajectories of the amide-I excitations seen in Fig. 4 correspond to the motion of a Brownian particle 


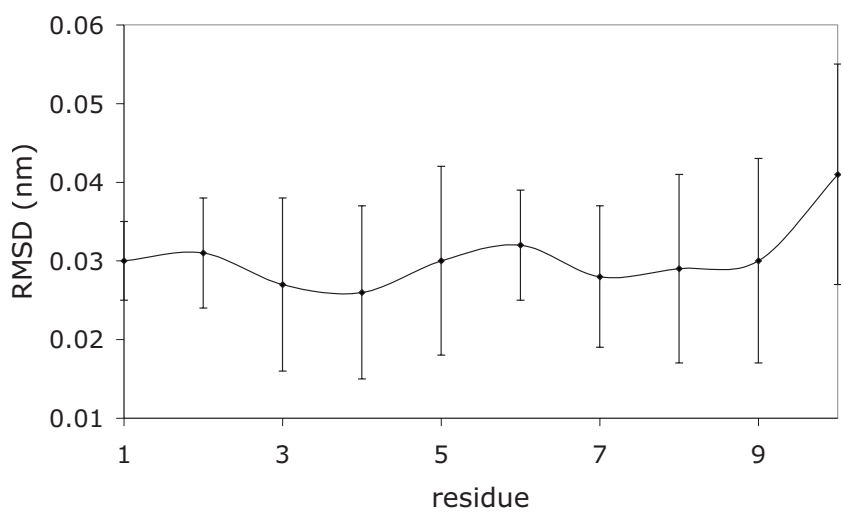

FIG. 5. RMSF of the $\alpha$ carbons of deca-alanine plotted as a function of residue number, averaged over 20 trajectories corresponding to $\chi=100 \mathrm{pN}$; error bars represent standard deviation. RMSF was determined by the $g_{-} r m s f$ GROMACS analysis tool (Refs. 8 and 9).

rather than to that of a coherent excitation or soliton. This is in contrast to what has been seen in simulations run at low temperature, which used the same scheme to determine intersite transfer of the amide-I excitation. That is, when thermal energy was weak by comparison to the soliton binding energy (defined below), a coherentlike excitation could be found. ${ }^{15}$ However because the strength of the approach taken here lies in its computation of equilibrium averages consistent with correct mixed quantum-classical statistics, rather than in its rigorous time propagation, a detailed understanding of the dynamics of a vibrational excitation will depend upon the future development of methods more suitable for treating time dependence.

\section{CONCLUSION}

The results just presented raise the possibility that, following ATP hydrolysis, the vibrational energy created could instigate biologically significant secondary-structural transitions within a protein system. Various examples are known of minor conformational changes occurring within $\alpha$-helical protein segments that are key to large-scale biomolecular events. For example, a helical/unfolded double-state system has been reported to be associated with the hydrolysis of ATP in actin. ${ }^{27,28}$ Additional examples include the highly conserved relay helices of kinesin and of myosin, which are believed to undergo conformational transitions responsible for instigating the power strokes in these two motor proteins following ATP hydrolysis. ${ }^{29}$ Such a conformational change of a protein arising from a localized vibrational mode would represent a specifically quantum-mechanical effect, since previous studies have demonstrated that in the case of a system of classical oscillators, vibrational energy becomes quickly delocalized. ${ }^{15,17,30-32}$

The values of $\chi$ for which an amide-I state was found to significantly impact helical content, in this study, are greater than existing estimates. However, the same types of terms arise in the interaction energy term, Eq. (2), for the $\mathrm{NH}$ stretch as for the amide-I vibration, and thus results are expected to be similar when considering the $\mathrm{NH}$ vibration which has previously been proposed in the literature to be the molecular vibration key to protein energy transfer. ${ }^{11,33-36}$ The parameter of nonlinearity $\chi$ is proportional to the square root of the binding energy, which is given by the energetic difference between the lowest energy state and that which is completely delocalized. ${ }^{11,37}$ It has been estimated experimentally that the binding energy of the $\mathrm{NH}$ stretch is about 20 times as large as that of the amide-I excitation, ${ }^{34}$ and so the value of $\chi$ corresponding to the $\mathrm{NH}$ stretch may be expected to be four to five times larger than that for the amide-I vibration. In this context, it is reasonable to estimate that $\chi$ may be as large as several hundred piconewton.

In summary, we propose that a vibrationally excited state, created following ATP hydrolysis, could work toward driving the system along a reaction pathway corresponding to a structural transition to a more helical state, by effectively subtracting the energy of the vibrational excitation directly from the activation energy required for this transition. Once the reaction product, i.e., the more helical structure, has been created, there are two thermodynamic possibilities. It is possible that in the presence of the hydrolyzed nucleotide, the helical form is more stable than the less structured form, which was thermodynamically favored before hydrolysis. However, a second distinct possibility is that the less structured form remains favored thermodynamically, in which case after some time the structure would be expected to return to the less helical form. Speculatively, reversions of this type may be important stages in certain chemomechanical cycles of proteins, such as corresponding, for example, to the rescue of microtubules, i.e., their growth subsequent to a period of catastrophic shrinkage, for which a satisfactory explanation is currently lacking. ${ }^{38}$

\section{ACKNOWLEDGMENTS}

We thank Tyler Luchko for proposing deca-alanine as a model system. H.F. is grateful for support from the Portuguese Foundation for Science and Technology (FCT) under Grant No. SFRH/BPD/41143/2007.

\section{APPENDIX}

In this appendix we review how the finite-temperature equations of motion, Eqs. (3)-(6), were arrived at. In the current study they have been used because they provide a proper representation of the quantum statistics of the amide-I states, as well as a proper representation of the classical statistics of the atoms in the protein. The problems that arise in a dynamical description of a mixed quantum-classical system when the classical part is coupled to a classical bath have been pointed out in a number of publications, ${ }^{30,39,40}$ and are described briefly below. Equations (3)-(6) used here constitute one solution to these problems.

The first study of the effect of thermal agitation on the Davydov/Scott system was made by Lomdahl and Kerr. ${ }^{32}$ They started from the Ehrenfest equations of motion for an amide-I excitation in a one-dimensional chain, 


$$
\begin{gathered}
i \hbar \frac{d \varphi_{n}}{d t}=\varepsilon \varphi_{n}-V\left(\varphi_{n-1}+\varphi_{n+1}\right)+\chi\left(u_{n+1}-u_{n-1}\right) \varphi_{n}, \\
M \frac{d^{2} u_{n}}{d t^{2}}=\chi\left(\left|\varphi_{n+1}\right|^{2}-\left|\varphi_{n-1}\right|^{2}\right)+\kappa\left(u_{n+1}-u_{n-1}-2 u_{n}\right),
\end{gathered}
$$

where $J_{n m}=V$, i.e., only nearest-neighbor dipole-dipole interactions are considered, $\kappa$ is the elasticity of the lattice, and where the atomic Hamiltonian of Eqs. (3)-(6) was substituted by the phonon Hamiltonian: $\hat{H}_{p h}=\frac{1}{2} \sum_{n=1}^{N}\left[\kappa\left(\hat{U}_{n}\right.\right.$ $\left.\left.-\hat{U}_{n-1}\right)^{2}+\frac{\hat{P}_{n}^{2}}{M}\right]$.

Equations (A1) and (A2) provide an accurate representation of the dynamics of a mixed quantum-classical system when the quantum particle remains in a single energy state. When quantum transitions can occur, another method, the surface hopping method, has been proposed. ${ }^{41}$ However, both Ehrenfest and surface hopping dynamics lead to incorrect thermal equilibrium properties in the case of a mixed quantum-classical system. ${ }^{15,30,40,42}$ On the other hand, the equations of motion we use Eqs. (3)-(6) not only allow for amide-I quantum transitions but also make sure that this quantum subsystem remains correctly thermalized in the process.

To understand the full problem, let us start by considering the classical thermalization of Ehrenfest Eqs. (A1) and (A2), as implemented in Ref. 32,

$$
i \hbar \frac{d \varphi_{n}}{d t}=\varepsilon \varphi_{n}-V\left(\varphi_{n-1}+\varphi_{n+1}\right)+\chi\left(u_{n+1}-u_{n-1}\right) \varphi_{n},
$$

$$
\begin{aligned}
M \frac{d^{2} u_{n}}{d t^{2}}= & \chi\left(\left|\varphi_{n+1}\right|^{2}-\left|\varphi_{n-1}\right|^{2}\right)+\kappa\left(u_{n+1}-u_{n-1}-2 u_{n}\right)+F_{n}(t) \\
& -\Gamma \frac{d u_{n}}{d t},
\end{aligned}
$$

where the only difference with respect to Eqs. (A1) and (A2) is that stochastic forces $F_{n}$ and damping terms $\Gamma \frac{d u_{n}}{d t}$ have been added to the equation of motion of the classical degrees of freedom, Eq. (A2). When these latter terms obey the fluctuation-dissipation theorem, i.e., when $\left\langle F_{n}(t) F_{m}\left(t^{\prime}\right)\right\rangle$ $=2 M \Gamma k_{B} T \delta_{n m} \delta\left(t-t^{\prime}\right)$, the corresponding Langevin equations are known to lead to the correct thermalization of all classical systems. On the other hand, it can be shown that such a thermalization of a mixed quantum-classical system leads to a classical behavior of the quantum subsystem. Indeed, as pointed out in Ref. 15, Eqs. (A3) and (A4) are identical to the equations of motion of the fully classical system that is obtained when the variables $\varphi_{n}$ are taken to be classical amplitudes instead of probability amplitudes. Such classical equations lead of course to a diffusion in the total phase space of the system that obeys classical statistics. Put in another way, this means that the equilibrium average of any classical variable $B\left(\left\{u_{n}\right\},\left\{\varphi_{n}\right\}\right)$, dependent on the classical displacements $u_{n}$ and on the classical amplitudes $\varphi_{n}$, as obtained from sufficiently long dynamical simulations of Eqs. (A3) and (A4), is equal to the thermal average that can be obtained in a completely independent Monte Carlo simulation of the following fully classical expression, ${ }^{31}$

$$
\left\langle\left\langle B\left(\left\{u_{n}\right\},\left\{\varphi_{n}\right\}\right)\right\rangle\right\rangle=\frac{\int_{2 N \text { unit sphere }}\left\{d \varphi_{n}^{r}\right\}\left\{d \varphi_{n}^{i}\right\} \int\left\{d u_{n}\right\} e^{-\beta H\left(\left\{u_{n}\right\}\left\{\varphi_{n}\right\}\right)} B\left(\left\{u_{n}\right\},\left\{\varphi_{n}\right\}\right)}{\int_{2 N \text { unit sphere }}\left\{d \varphi_{n}^{r}\right\}\left\{d \varphi_{n}^{i}\right\} \int\left\{d u_{n}\right\} e^{\left.-\beta H\left(\left\{u_{n}\right\} \varphi_{n}\right\}\right)}} .
$$

Here $\langle\langle\cdots\rangle\rangle$ stands for equilibrium average, $\beta=1 / k_{B} T$, and $H\left(\left\{u_{n}\right\},\left\{\varphi_{n}^{r}\right\}\left\{\varphi_{n}^{i}\right\}\right)$ is the fully classical Hamiltonian whose dependence on the classical displacements $u_{n}$ and on the real and imaginary parts $\left\{\varphi_{n}^{r}\right\},\left\{\varphi_{n}^{i}\right\}$, respectively, of the classical amplitudes $\varphi_{n}$ is formally identical to the dependence of the mixed-quantum classical Hamiltonian on displacements $u_{n}$ and on probability amplitudes $\varphi_{n}$. Thus, both Eq. (A5) and Eqs. (A3) and (A4) pertain to descriptions of the fully classical Davydov/Scott system. On the other hand, as is pointed out in Ref. 15, the correct expression for the equilibrium average of a mixed quantum-classical variable $\hat{B}\left(\left\{u_{n}\right\},\left\{\hat{a}_{n}^{\dagger}\right\},\left\{\hat{a}_{n}\right\}\right)$ dependent on the classical displacements $u_{n}$ and on the quantum operators $\left(\left\{\hat{a}_{n}^{\dagger}\right\},\left\{\hat{a}_{n}\right\}\right)$ is

$$
\left\langle\left\langle\hat{B}\left(\left\{u_{n}\right\},\left\{\hat{a}_{n}^{\dagger}\right\},\left\{\hat{a}_{n}\right\}\right)\right\rangle\right\rangle=\frac{\int\left\{d u_{n}\right\} e^{-\beta H_{a t}} \sum_{j=1, N} e^{-\beta E_{j}}\left\langle\phi_{j}|\hat{B}| \phi_{j}\right\rangle}{\int\left\{d u_{n}\right\} e^{-\beta H_{a t}} \sum_{j=1, N} e^{-\beta E_{j}}},
$$

where $E_{j}$ is the energy of eigenstate $\phi_{j}$ of the (quantum) amide-I vibration for a conformation $\left\{u_{n}\right\}$ of the (classical) lattice and the summation is over all $N$ eigenstates of the quantum particle. 
Equation (A6) expresses mathematically the quantum statistics rule that stipulates that the quantum states must be taken with all possible random phases so that ultimately only eigenstates contribute with a nonzero weight to the equilibrium averages while Eq. (A5) includes the contributions of all possible superpositions of eigenstates as well. The problem with equations of motion, Eqs. (A3) and (A4), is precisely that they allow the amide-I wave function to visit all possible superpositions of eigenstates, thus leading to classical statistics. In order to obey quantum statistics, a constraint should be imposed on the dynamics provided by Eqs. (A3) and (A4) to restrict the diffusion in quantum phase space in a suitable manner. The equations of motion used here, Eqs. (3)-(6), by coupling the time-independent Schrödinger equation for the amide-I vibrations to the classical equations of motions for the atoms in the protein, provide such a constraint and can indeed lead to the correct mixed quantumclassical thermal equilibrium averages. ${ }^{15,31}$

Finally, it should be stressed that the problem discussed here is absolutely general. It arises whenever any mixed quantum-classical system is coupled to a classical bath, ${ }^{40,42}$ and the equations of motion, Eqs. (3)-(6), used here are only one of the solutions that have been proposed. In the Car/ Parrinello method, ${ }^{43}$ the solution is to periodically bring down the electrons to the ground state, which is valid as long as the energetic difference of the first excited state with respect to the ground state is much higher than thermal energy $\left(k_{B} T\right)$. Another solution that has been implemented in a quantum-classical dimer was to introduce, in the Ehrenfest equations of motion, a term with a Lagrange multiplier to force the density matrix to tend to its exact analytical expression. ${ }^{39,44}$ Neither of these two solutions can be used in the case of the Davydov/Scott system. Indeed, as determined in a simulation of the thermal equilibrium properties of the full quantum Davydov/Scott system, ${ }^{45}$ the energy of the excited states of the amide-I vibration are only $11 k_{B}$ higher than the ground state; that is, above $11 \mathrm{~K}$, the excited states will have a high probability of being populated. This is why the Davydov soliton, which is the ground state of the system, is not thermally stable at biological temperatures. ${ }^{15,17}$ The second solution mentioned above is not feasible either because the Davydov/Scott system (and even more so the extension of it that we use here) is too complicated and an analytical expression for the partition function is not available. The equations of motion, Eqs. (3)-(6), are thus a third possible solution to the thermalization problem that is applicable even to this extended Davydov/Scott system and indeed to any other mixed quantum-classical system. However it is not without its own limitations. One limitation is that Eqs. (3)-(6) are only strictly valid when the amide-I vibration adapts very quickly to the changes in the atomic coordinates; ${ }^{15}$ a second limitation of Eqs. (3)-(6) is that they do not keep any memory of the phases of the wave functions and thus cannot describe quantum effects such as (de)coherence and entanglement. However, we think that, in the noisy environment in which proteins work, such quantum effects do indeed fade out very fast and do not play a role in the energy-transfer processes that we are interested in. One other quantum effect that we are interested in, that is, amide-I localization in a few amino acids, however, is important. Indeed, localization has been shown to be a truly quantum effect since the fully classical system, represented by dynamical Eqs. (A3) and (A4) and by the corresponding equilibrium Eq. (A5), does lead to delocalized amide-I vibrations, ${ }^{31,32}$ while the exact mixed quantum-classical system (as well as the full quantum system) leads to localized amide-I vibrations. ${ }^{15,16,30,45}$ Thus, while for other mixed quantum-classical systems the surface hopping method may provide a reasonably good approximation of the thermal equilibrium properties, ${ }^{40}$ if we want to have the correct localization of the amide-I state in the (extended) Davydov/ Scott system, we need to use other equations of motion, such as those we use here.
${ }^{1}$ A. S. Davydov, J. Theor. Biol. 38, 559 (1973).

${ }^{2}$ A. S. Davydov, Solitons in Molecular Systems (D. Reidel, Dordrecht, 1985).

${ }^{3}$ P. S. Lomdahl, S. P. Layne, and I. J. Bigio, Los Alamos Sci. 10, 2 (1984).

${ }^{4}$ P. Hamm, J. Biol. Phys. 35, 17 (2009).

${ }^{5}$ V. Botan, E. H. Backus, R. Pfister, A. Moretto, M. Crisma, C. Toniolo, P. H. Nguyen, G. Stock, and P. Hamm, Proc. Natl. Acad. Sci. U.S.A. 104, 12749 (2007).

${ }^{6}$ L. Cruzeiro, J. Phys.: Condens. Matter 17, 7833 (2005).

${ }^{7}$ L. Cruzeiro, J. Chem. Phys. 123, 234909 (2005).

${ }^{8}$ E. Lindahl, B. Hess, and D. van der Spoel, J. Mol. Model. 7, 306 (2001).

${ }^{9}$ D. Van Der Spoel, E. Lindahl, B. Hess, G. Groenhof, A. E. Mark, and H. J. Berendsen, J. Comput. Chem. 26, 1701 (2005).

${ }^{10}$ V. Hornak, R. Abel, A. Okur, B. Strockbine, A. Roitberg, and C. Simmerling, Proteins 65, 712 (2006).

${ }^{11}$ A. C. Scott, I. J. Bigio, and C. T. Johnston, Phys. Rev. B 39, 12883 (1989).
${ }^{12}$ D. A. Case, T. A. Darden, T. E. Cheatham III, C. L. Simmerling, J. Wang, R. E. Duke, R. Luo, K. M. Merz, D. A. Pearlman, M. Crowley, R. C. Walker, W. Zhang, B. Wang, S. Hayik, A. Roitberg, G. Seabra, K. F. Wong, F. Paesani, X. Wu, S. Brozell, V. Tsui, H. Gohlke, L. Yang, C. Tan, J. Mongan, V. Hornak, G. Cui, P. Beroza, D. H. Mathews, C. Schafmeister, W. S. Ross, and P. A. Kollman, AMBER 9, University of California, San Francisco, 2006.

${ }^{13}$ N. A. Nevskaya and Y. N. Chirgadze, Biopolymers 15, 637 (1976).

${ }^{14}$ S. Krimm and J. Bandekar, Adv. Protein Chem. 38, 181 (1986).

${ }^{15}$ L. Cruzeiro-Hansson and S. Takeno, Phys. Rev. E 56, 894 (1997).

${ }^{16}$ L. Cruzeiro-Hansson and V. M. Kenkre, Phys. Lett. A 203, 362 (1995).

${ }^{17}$ L. Cruzeiro-Hansson, Phys. Lett. A 223, 383 (1996).

${ }^{18}$ D. R. Roe, A. Okur, L. Wickstrom, V. Hornak, and C. Simmerling, J. Phys. Chem. B 111, 1846 (2007).

${ }^{19}$ W. D. Cornell, P. Cieplak, C. I. Bayly, I. R. Gould, K. M. Merz, 
Jr., D. M. Ferguson, D. C. Spellmeyer, T. Fox, J. W. Caldwell, and P. A. Kollman, J. Am. Chem. Soc. 117, 5179 (1995).

${ }^{20}$ J. Wang, P. Cieplak, and P. A. Kollman, J. Comput. Chem. 21, 1049 (2000).

${ }^{21}$ W. L. Jorgensen, J. Chandrasekhar, J. D. Madura, R. W. Impey, and M. L. J. Klein, J. Chem. Phys. 79, 926 (1983).

${ }^{22}$ D. L. Mobley, J. D. Chodera, and K. A. Dill, J. Chem. Phys. 125, 084902 (2006).

${ }^{23}$ B. Hess, H. Bekker, H. J. C. Berendsen, and J. G. E. M. Fraaije, J. Comput. Chem. 18, 1463 (1997).

${ }^{24}$ J. Edler and P. Hamm, J. Chem. Phys. 117, 2415 (2002).

${ }^{25}$ W. Kabsch and C. Sander, Biopolymers 22, 2577 (1983).

${ }^{26}$ L. Brizhik, A. Eremko, B. Piette, and W. Zakrzewski, Phys. Rev. E 70, 031914 (2004).

${ }^{27}$ J. W. Chu and G. A. Voth, Proc. Natl. Acad. Sci. U.S.A. 102, 13111 (2005).

${ }^{28}$ J. Pfaendtner, D. Branduardi, M. Parrinello, T. D. Pollard, and G. A. Voth, Proc. Natl. Acad. Sci. U.S.A. 106, 12723 (2009).

${ }^{29}$ R. D. Vale and R. A. Milligan, Science 288, 88 (2000).

${ }^{30}$ L. Cruzeiro-Hansson, Europhys. Lett. 33, 655 (1996).
${ }^{31}$ L. Cruzeiro-Hansson, Physica D 68, 65 (1993).

${ }^{32}$ P. S. Lomdahl and W. C. Kerr, Phys. Rev. Lett. 55, 1235 (1985).

${ }^{33}$ D. M. Alexander, Phys. Rev. Lett. 54, 138 (1985).

${ }^{34}$ D. M. Alexander and J. A. Krumhansl, Phys. Rev. B 33, 7172 (1986).

${ }^{35}$ G. Careri, U. Buontempo, F. Galluzzi, A. C. Scott, E. Gratton, and E. Shyamsunder, Phys. Rev. B 30, 4689 (1984).

${ }^{36}$ A. Scott, Phys. Rep. 217, 1 (1992).

${ }^{37}$ L. Cruzeiro-Hansson and V. M. Kenkre, Phys. Lett. A 190, 59 (1994).

${ }^{38}$ R. H. Wade, Mol. Biotechnol. 43, 177 (2009).

${ }^{39}$ G. P. Tsironis and V. M. Kenkre, Phys. Lett. A 127, 209 (1988).

${ }^{40}$ J. R. Schmidt, P. V. Parandekar, and J. C. Tully, J. Chem. Phys. 129, 044104 (2008).

${ }^{41}$ J. C. Tully, J. Chem. Phys. 93, 1061 (1990).

${ }^{42}$ F. Mauri, R. Car, and E. Tosatti, Europhys. Lett. 24, 431 (1993).

${ }^{43}$ R. Car and M. Parrinello, Phys. Rev. Lett. 55, 2471 (1985).

${ }^{44}$ V. M. Kenkre and H.-L. Wu, Phys. Lett. A 135, 120 (1989).

${ }^{45}$ X. Wang, D. W. Brown, and K. Lindenberg, Phys. Rev. Lett. 62, 1796 (1989). 